\title{
ASPECTOS ECOLÓGICOS DE FUNGOS MICORRÍzICOS VESÍCULO-ARBUSCULARES DA MATA TROPICAL ÚMIDA DA ILHA DO CARDOSO, SP, BRASIL'
}

\author{
Sandra F. B. Trufem ${ }^{2}$
}

Recebido em 28-3-90. Aceito em 10-1-91.

RESUMO - No período de agosto/1984 a maio/1987, em nove ocasiões, foram coletadas 300 amostras de solo da rizosfera de 35 espécies de plantas nativas da mata tropical úmida do Parque Estadual da Ilha do Cardoso, SP, Brasil, para a investigação da ocorrência de fungos micorrízicos vesículo-arbusculares (MVA) e a obtençấo de dados sobre aspectos ecológicos desses microrganismos. Foram verificados 35 taxa de fungos MVA, sete dos quais espécies novas. Os esporos que ocorreram em maior quantidade foram os de Acaulospora foveata Trappe \& Janos, Acaulospora scrobiculata Trappe, Glomus etunicatum Becker \& Gerd.. Glomus geosporum (Nicol. \& Gerd.) Walker, Glomus macrocarpum Tul. \& Tul. e Glomus microcarpum Tul. \& Tul. As plantas cujas rizosferas apresentaram maior número de esporos de fungos MVA foram as de Blechnum serrulatum L.C. Rich., Euterpe edulis. Mart, Neomarica caerulia Sprague, Geonoma elegans Mart. e Piper aduncum L. A maior diversidade de espécies de fugnos MVA ocorreu nas rizosferas de Aphellandra sp., Geonoma elegans Mart e Leandra barbinervis Cogn. Verificouse que houve predominância de espécies de fungos micorrízicos vesículo-arbusculares clamidospóricas sobre as azigospóricas, bem como que a quantidade de esporos em uma dada rizosfera está diretamente relacionada com a diversidade. Glomus monosporum Gerd. \& Trappe e as espécies de Sclerocystis podem ser apontadas como características do ecossistema estudado, que apresentou a média de 50,04 esporos/ $100 \mathrm{~g}$ de solo

Palavras-chave: ecologia de fungos MVA; floresta tropical úmida: Brasil

ABSTRACT - From August/1984 to May/1987, 300 soil samples were collected from the rhizospheres of 35 species of native plants of Parque Estadual da Ilha do Cardoso, SP, Brazil, in a wet tropical forest, to investigate the occurrence and some ecological aspects of vesicular-arbuscular mycorrhizal (VAM) fungi. Thirty five taxa of VAM fungi were reported, seven of them as new species. The most numerous VAM fungal spores belong to Acaulospora foveata Trappe \& Janos, Acaulospora scrobiculata Trappe. Glomus etunicatum Becker \& Gerd., Glomus geosporum (Nicol. \& Gerd.) Walker, Glomus macrocarpum Tul. \& Tul. and Glomus microcarpum Tul. \& Tul. The rhizospheres that presented the highest number of VAM fungi spores were Blechnum serrulatum L.C. Rich., Euterpe edulis Mart.,

1 - Parte da Tese de Doutorado defendida no Inst. Biociéncias. Univ. S, Paulo

2 - Instituto de Botanica. Caixa Postal 4005. 01051. Sao Paulo. SP, Brasil. Bolsista do $\mathrm{CNPq}$ 
Neomarica caerulia Sprague and Piper aduncum L. The highest diversity of VAM fungi species occurred in the rhizospheres of Aphellandra sp., Geonoma elegans Mart. and Leandra barbinervis Cogn. It was observed that clamydosporic species of vesicular-arbuscular mycorrhizal fungi were more numerous than azygosporic species; the number of spores is correlated with the diversity in a rhizosphere. Glomus monosporum Gerd. \& Trappe and Sclerocystis species are characteristic of the studied ecosystem, that presented the average of 50,04 spores $/ 100 \mathrm{~g}$ of soil.

Key-words: VAM fungal ecology; wet tropical forest; Brazil.

\section{Introduçāo}

Os fungos micorrízicos vesículo-arbusculares (MVA) ocorrem nas raízes da maioria das plantas terrestres (Gerdemann, 1968), sendo exceçâo a sua ausência. A associação fungo MVA-planta hospedeira distingue-se pelo caráter simbiotrófico: a planta cede ao fungo elementos produzidos pela fotossíntese e o microrganismo transfere ao vegetal água e elementos minerais que foram absorvidos da solução do solo pelas hifas que, por sua vez, colonizam o solo para além da zona de depleção das raízes (Cabala-Rosand \& Dias, 1986).

Os fungos MVA ainda têm sido referidos como capazes de conferir à planta que os abriga algum tipo de tolerância/resistência contra o ataque por agentes fitopatogênicos, como outros fungos e nematóides (Dehne, 1982), com menores agressões ao meio ambiente, no que se refere a agentes de controle fitossanitário.

Regiōes desérticas têm sido revegetadas com maior eficiência com a participação de fungos MVA (Reeves et al., 1979) bem como áreas sujeitas a erosões, deslizamentos e outros tipos de impactos ambientais, como chuvas ácidas e metais pesados (Gildon \& Tinker, 1983 a,b; Day et al., 1987).

Este trabalho teve como objetivos relacionar as espécies de fungos MVA e a apresentar considerações ecológicas desses organismos em rizosferas de plantas nativas de mata tropical úmida.

\section{Material e Métodos}

No período de agosto/1984 a maio/1987, em nove ocasiōes, foram coletadas 300 amostras de solo da rizosfera de 35 espécies de plantas nativas de mata, no Parque Estadual da Ilha do Cardoso, SP, Brasil (250 05' e 250 15' S; $47^{\circ} 53^{\prime}$ e $48^{\circ} 06^{\prime} \mathrm{W}$ ), para a investigação da ocorrência de fungos MVA e a obtenção de dados sobre aspectos ecológicos desses organismos. A relação das plantas estudadas, bem como as respectivas famílias e datas de coletas, encontram-se expressas na Tabela 1. 
Tabela 1 - Plantas cujas rizosferas foram estudadas, respectivas famúlias e datas em que foram coletadas.

Planta hospedeira

Família

Data de Coleta

Anemia sp. . . . . . . . . . . . . . . Schizaeaceae

Aphellandra $\mathrm{sp} . \ldots \ldots \ldots$. . . . . . Acanthaceae

Araceae

Araceae

Bauhinia sp. . . . . . . . . . . . Leguminosae

Begonia sp. . . . . . . . . . . . Begoniaceae

Blechnum serrulatum L.C. Rich. . . . . . Polypodiaceae

Centella asiatica (L.) Urban . . . . . . . . . U Umbelliferae

Clidemia hirta D. Don. . . . . . . . . . . Melastomataceae

Coccocypselum cordifolium Ness. \& Mart. . . Rubiaceae

Compositae . . . . . . . . . . . . . Compositae

Cyperus sp. . . . . . . . . . . . Cyperaceae

Dichorisandra thyrsiflora Mikan. . . . . . . Commelinaceae

Euterpe edulis Mart.. . . . . . . . . . . Palmae

Geonoma elegans Mart. . . . . . . . . . Palmae

Inga affinis D.C. . . . . . . . . . Leguminosae

Leandra barbinervis Cogn. . . . . . . . . . . Melastomataceae

Maranta sp............... Marantaceae

Miconia inaequidens Naud. . . . . . . . . . Melastomataceae

Neomarica caerulia Sprague. . . . . . . . . Iridaceae

Parapiptadenia rigida (Bentham) Brenan. Leguminosae

Paspalum sp............... Gramineae

Piper aduncum L... . . . . . . . . . Piperaceae

Psychotria nuda (Mart.) Wawra. . . . . . Rubiaceae

Psychotria tristicula Standal. . . . . . . . Rubiaceae

Quesnelia arvensis (Vell.) Mez. . . . . . . Bromeliaceae

Selaginella sp............... Sellaginelaceae

Spigelia humboldtiana Cham. \& Schulcht. . . Loganiaceae

Tibouchina sp................ Melastomataceae

Planta n. 19a.

XII/1984-II/1985

XII/1984-II/1985-

IX/1985-VI/1986

II/ 1985

II/ 1985

II/ 1985

III/1985

II/ 1985-III/ 1986

IX/1985

V/1987

III/ 1986

VI/1985-VI/ 1986

XII/1984-VI/ 1985

XII/1984-II/1985-

IX/1985-III/1986-

VI/1986-V/1987

XII/1984-II/1985-

VI/1985-IX/1985-

V/1987

II/1985

VI/1985-IX/1985-

VI/1986

IX/1985-III/1986-

VI/1986-VI/1986-

V/1987

VI/ 1986

XII/1984-XII/ 1984

VI/1985

VIII/1986

III/1986

VI/1986-V/1987

II/1985

III/1986

IX/1985

II/1985

II/1985

III/1986

XII/1984

VI/1985

IX/1985

VI/1985

XII/1984

VI/1985

VI/1985 
Para cada espécie de planta em cada ocasião de coleta, foram amostradas cinco rizosferas, provenientes de plantas distintas, para constituir uma amostra composta. As amostras de solo foram coletadas até $15 \mathrm{~cm}$ de profundidade e acondiconadas em sacos plásticos transparentes, armazenados em refrigeração $\left(4-8^{\circ} \mathrm{C}\right)$ até o momento do processamento.

As amostras compostas foram preparadas de acordo com a técnica do peneiramento em via úmida (Gerdemann \& Nicolson, 1963) com o uso de peneiras com malhas de $750,350,105$ e $53 \mu \mathrm{m}$ diâm.

Os esporos, sob estereomicroscópio, foram separados, distribuídos em grupos de acordo com suas semelhanças morfológicas, contados e a seguir montados entre lâmina e lamínula com água e/ou resina de álcool polivinílico e lactofenol - resina PVL (Walker, 1979), para observação ao microscópio óptico e estudos taxonômicos.

Amostras de solo de mata foram coletadas e encaminhadas para análise dos elementos químicos.

\section{Resultados e Discussão}

Foram verificadas 35 espécies de fungos MVA, a saber: Acaulospora appendicula Spain, Sieverding \& Schenck, A. elegans Trappe \& Gerd., A. foveata Trappe \& Janos, A. lacunosa Morton, A. laevis Gerd. \& Trappe, A. mellea Spain \& Schenck, A. scrobiculata Trappe, A. spinosa Walker \& Trappe, A. tuberculata Janos \& Trappe, três espécies novas de Acaulospora, Entrophospora infrequens (Hall) Ames \& Schneider, Gigaspora gigantea Nicol. \& Gerd., Glomus claroideum Schenck \& Smith. G. etunicatum Becker \& Gerd., G. fasciculatum (Thaxter) Gerd. \& Trappe emend. Walker \& Koske, G. geosporum (Nicol. \& Gerd.) Walker, G. intraradices Schenck \& Smith, $G$. invermaium Hall, G. macrocarpum Tul. \& Tul., G. microcarpum Tul. \& Tul., G: monosporum Gerd. \& Trappe, G. pallidum Hall, duas espécies novas de Glomus, Sclerocystis clavispora Trappe, S. coremioides Berk. \& Br., S. microcarpus Iqbal \& Bushra, Sclerocystis sp. 1, Scutellospora aurigloba (Hall) Walker \& Sanders, S. calospora (Nicol. \& Gerd.) Walker \& Sanders, S. erythropa (Koske \& Walker) Walker \& Sanders, S. nigra (Redhead) Walker \& Sanders e uma espécie nova de Scutellospora.

A distribuição dessas espécies em função da rizosfera das plantas hospedeiras encontra-se expressa nas Tabelas 2 a 6.

Os dados de análise dos elementos químicos do sulo encontram-se expressos na Tabela 7.

A análise das Tabelas 2 a 5 evidencia que as espécies mais abundantes de fungos MVA foram G. macrocarpum (963 esporos), A. foveata (363 esporos), A. scrobiculata e $G$. geosporum (150 esporos cada), G. etunicatum (147 esporos), G. microcarpum (126 esporos), A. mellea (108 esporos) e assim por 
diante, até chegar-se a espécies que foram verificadas com apenas seis esporos (A. elegans, A. lacunosa, G. claroideum, G. invermaium) ou até mesmo três esporos (A. foveta, $G$. gigantea).

A Tabela 2 demonstra que o gênero Acaulospora ocorreu com 882 esporos, distribuídos em 12 espécies. A Tabela 3 evidencia Entrophospora infrequens, com 48 esporos, Gigaspora gigantea com apenas três esporos e Sclerocystis, com 108 esporocarpos, em quatro espécies. O gênero Glomus (Tabela 4) apresentou 1569 esporos, distribuídos em 12 espécies, e, finalmente, o gênero Scutellospora (Tabela 5), com 228 esporos, distribuídos em cinco espécies. Condensando-se esses dados (Tabela 6), percebe-se que as espécies de fungos MVA azigospóricas fizeram-se presentes com 1161 esporos, portanto, $39,25 \%$ do total de esporos observados, enquanto que as espécies clamidospóricas ocorreram com 1797 esporos/esporocarpos, representando 60,75\% do total de esporos observados.

Os diversos trabalhos realizados com fungos MVA em ecossistemas de mata, insistem em enfatizar a ocorrência natural desses organismos (Redhead, 1968; Hogberg, 1982; St. John, 1980a, 1980b; Santos \& Vinha, 1982; Bononi \& Trufem, 1983), o que é confirmado no presente estudo.

Por outro lado, a literatura tem sido repetitiva em reafirmar que os fungos MVA não apresentam especificidade de hospedeiro (Kruckelmann, 1975; Mosse et al.,1981), ao lado de autores que advogam a existência de preferências de algumas espécies de fungos MVA por determinados hospedeiros (Bononi \& Trufem, 1983, Barea et al., 1984). O presente trabalho confirma os dados desses autores, demonstrando que há espécies de fungos MVA que ocorreram em maior diversidade de rizosferas, o que sugere menor especificidade destas em relação aos hospedeiros, e/ou maior suscetibilidade das plantas à micorrização. No caso de fungos MVA com ampla distribuição em diferentes rizosferas, citam-se as 12 espécies verificadas de Acaulospora (Tabela 2), que ocorreram em 26 diferentes espécies de plantas hospedeiras, sendo que $A$. foveata ocorreu em 16, A. spinosa em sete, A. appendicula, A. mellea e $A$. scrobiculata em cinco e assim por diante. As 12 espécies de Glomus (Tabela 4) ocorreram em 29 diferentes rizosferas, sendo que $G$. macrocarpum ocorreu em 21, G. monosporum em oito, G. microcarpum em sete, e assim por diante. As quatro espécies de Sclerocystis (Tabela 3) ocorreram em nove diferentes rizosferas e as cinco espécies de Scutellospora (Tabela 5) ocorreram em 13 diferentes espécies de rizosferas, sendo que $S$. calospora ocorreu em seis delas.

No que se refere à diversidade de esporos em função da rizosfera de uma mesma planta hospedeira, verificou-se (Tabela 6) que G. elgans, Aphellandra sp., L. barbinervis apresentaram, respectivamente, 13, dez e nove diferentes espécies de fungos MVA em suas rizosferas, existindo também as rizosferas que apresentaram apenas uma espécie de esporo de fungo MVA (Selaginella sp), ou duas (C. asiatica, $P$. rigida, $S$. humboldtiana, entre outras). 
Tabela 2: Distribuição de esporos de Acaulospora em função das rizosferas das plantas hospedeiras estudadas.

Plantas

hospedeiras

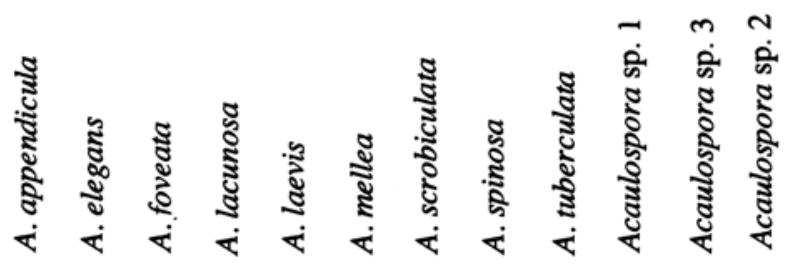

A B

Anemia sp.

Aphellandra sp.

Araceae

Begonia sp.

B. serrulatum

C. asiatica

C. hirta

C. cordifolium

Compositae

Cyperus sp.

D. thyrsiflora

E. edulis

G. elegans

I. affinis

L. barbinervis

Maranta sp.

N. caerulia

Paspalum sp.

$P$. aduncum

$P$. nuda

S. humboldtiana

Tibouchina sp.

$19 \mathrm{~b}$

23

25 b

26

- -3 - 3 - -1 -

$--12-9--6--3-304$

$-\quad 18--\quad-\quad-\quad-\quad-\quad-\quad-181$

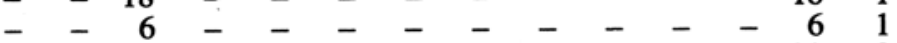

$-\quad 180--12-\quad-\quad-\quad-\quad-1922$

$-\quad 9--$ - - - - - 91

$---6----69-69-752$

$-------6--6-122$

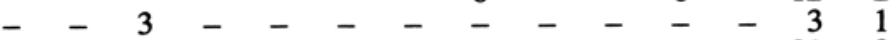

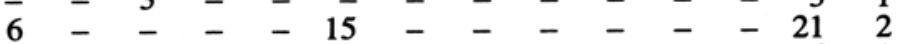

$--6---------61$

$6-27---6---\quad-393$

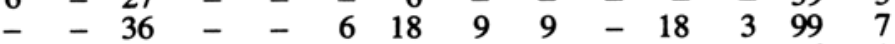

$-\quad 6--\quad-\quad-\quad-\quad-\quad-61$

$-\quad 12----3-6-6274$

$-\quad-\quad-\quad-2118-18-2$ - -573

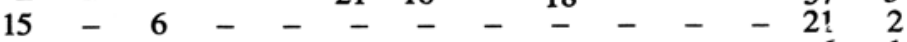

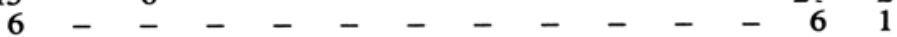

$-------9-9--182$

$-\quad 3-----6---31$

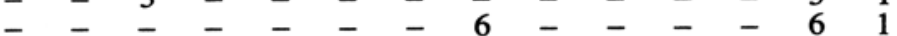

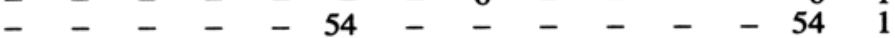

$-\quad-\quad-\quad-3018-\ldots-\quad-482$

$3-30--\quad-78--\quad-\quad-\quad-1113$

$-36------\quad-\quad-92$

$-3-\ldots-C-\quad-\quad-\quad-31$

\begin{tabular}{lrrrrrrrrrrrr}
\hline $\mathrm{C}$ & 36 & 6 & 363 & 6 & 9 & 108 & 150 & 57 & 96 & 15 & 27 & 9 \\
$\mathrm{D}$ & 5 & 2 & 16 & 1 & 1 & 5 & 5 & 7 & 3 & 2 & 3 & 2
\end{tabular}

Convenções:

A - n. total de esporos de fungos MVA em função da espécie de planta hospedeira que teve sua rizosfera investigada

B - n. total de espécies de fungo MVA que ocorreu em cada espécie de planta hospedeira que teve sua rizosfera investigada

C - n. total de esporos de fungo MVA de cada uma das espécies verificadas

D - n. total de rizosferas de cada uma das espécies de plantas investigadas, em que ocorreu cada um dos taxa de fungo MVA. 
Tabela 3: Distribuição das espécies de Entrophospora, Gigaspora e Sclerocystis nas rizosferas das plantas hospedeiras estudadas

\begin{tabular}{|c|c|c|c|c|c|c|c|c|c|c|c|c|}
\hline $\begin{array}{l}\text { Plantas } \\
\text { hospedeiras }\end{array}$ & 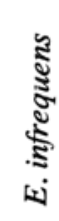 & $A$ & $B$ & 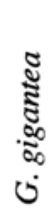 & $A$ & B & 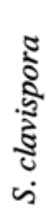 & 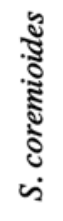 & 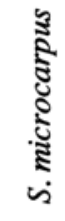 & 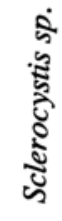 & A & B \\
\hline Anemia sp. & - & - & & 3 & 3 & 1 & - & - & - & - & - & - \\
\hline Aphellandra sp. & - & - & - & - & - & - & - & 3 & - & - & 3 & 1 \\
\hline Araceae & - & - & - & - & - & - & 3 & - & - & - & 3 & 1 \\
\hline C. cordifolium & - & - & - & - & - & - & - & - & - & 6 & 6 & 1 \\
\hline G. elegans & - & - & - & - & - & - & - & 3 & - & - & 3 & 1 \\
\hline Maranta sp. & - & - & - & - & - & - & - & - & 3 & - & 3 & 2 \\
\hline P. aduncum & - & - & - & - & - & - & - & - & 3 & 60 & 63 & 2 \\
\hline P. nuda & - & - & - & - & - & - & - & - & 15 & - & 15 & 1 \\
\hline S. humboldtiana & - & - & - & - & - & - & - & - & - & 3 & 3 & 1 \\
\hline Tibouchina sp. & - & - & - & - & - & - & - & 9 & - & - & 9 & 1 \\
\hline $19 \mathrm{~b}$ & 18 & 18 & 1 & - & - & _- & - & - & - & - & - & - \\
\hline 23 & 30 & 30 & 1 & - & - & - & - & - & - & - & - & - \\
\hline C & 48 & & & 3 & & & 3 & 15 & 2 & 69 & & \\
\hline D & 2 & & & 1 & & & 1 & 3 & 3 & 3 & & \\
\hline
\end{tabular}

Convenções:

A - n. total de esporos de fungos MVA em função da espécie de planta hospedeira que teve sua rizosfera investigada

B - n. total de espécies de fungo MVA que ocorreu em cada espécie de planta hospedeira que teve sua rizosfera investigada

C - n. total de esporos de fungo MVA de cada uma das espécies verificadas

D - n. total de rizosferas de cada uma das espécies de plantas investigadas, em que ocorreu cada um dos taxa de fungo MVA. 
Tabela 4: Distribuição das espécies đe Glomus em função das rizosferas das plantas hospedeiras estudadas.

Plantas hospedeiras
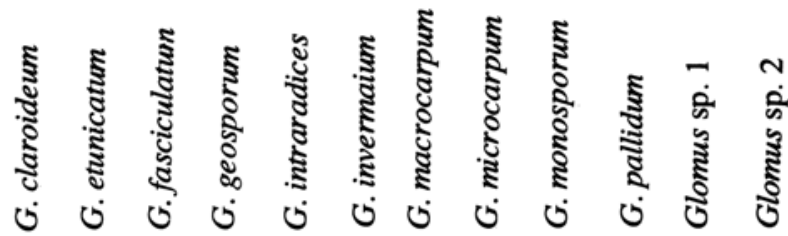

A B

Anemia sp.

- - - - - - 60

Aphellandra sp.

Araceae

Begonia sp.

C. asiatica

C. hirta

C. cordifolium

Compositae

D. thyrsiflora

E.edulis

G. elegans

I. affinis

L. barbinervis

Maranta sp.

N. caerulia

$P$. rigida

Paspalum sp.

$P$. aduncum

Selaginella $\mathrm{sp}$.

S. humboldtiana

Tibouchina sp.

19 a

$19 \mathrm{~b}$

$19 \mathrm{c}$

20

23

25 a

$25 \mathrm{~b}$

26

$$
-\quad-\quad-24
$$

$-\quad-\quad-\quad-18$

$-\quad-\quad-\quad-$

$-\quad-\quad--15$

- $-\quad-\quad-\quad 45$

- $-\quad-\quad-\quad 75$

- -

$-\quad-$

- -

$-\quad-\quad 375$

$-\overline{-} \overline{5}$

$15----33$

$-1 \overline{5}-\overline{-}-\overline{6}$

$6-$

$-9 \overline{0}$

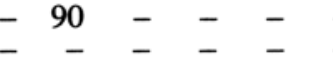

$-\quad-$

- $2 \overline{7}$

$-\quad-3--30$ $-\quad-3--3$

$-\quad-\quad-\quad-21$

$-\quad-\quad-\quad-\quad 183$

- - - - -30

- $-\quad-\quad-30$

$-\quad-\quad-\quad-\quad-\quad-\quad \frac{1}{9}-1$

- $-\quad-\quad-9$

$-\quad-\quad-\quad-30$

$-\quad-\quad-\quad-60$

$-\quad-\quad-60$

$24----105$

$-\quad-\quad-18$

$-$

51

- $-\quad-\quad-\quad-\quad-151$

$-\quad-39-573$

$\begin{array}{lllllll}- & - & 3 & - & - & 57 & 3\end{array}$

- $-\quad-\quad 30301$

$3015---252$

969

- 75

4

$-\quad-\quad-\quad-24$

3150

$\begin{array}{rrrrrrr}6 & 963 & 126 & 69 & 12 & 21 & 39 \\ 1 & 21 & 7 & 8 & 2 & 2 & 2\end{array}$

D

$\begin{array}{rr}6 & 147 \\ 1 & 4\end{array}$

27

Convenções:

A - n. total de esporos de fungos MVA em função da espécie de planta hospedeira que teve sua rizosfera investigada

B - n. total de espécies de fungo MVA que ocorreu em cada espécie de planta hospedeira que teve sua rizosfera investigada

C - n. total de esporos de fungo MVA de cada uma das espécies verificadas

D - n. total de rizosferas de cada uma das espécies de plantas investigadas, em que ocorreu cada um dos taxa de fungo MVA. 
Tabela 5: Distribuição das espécies de Scutellospora em função das rizosferas das plantas estudadas.

\begin{tabular}{|c|c|c|c|c|c|c|c|}
\hline $\begin{array}{l}\text { Plantas } \\
\text { hospedeiras }\end{array}$ & 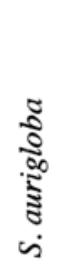 & 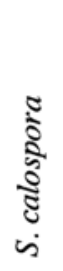 & 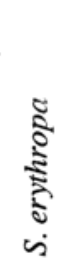 & $\underset{\check{D}}{\stackrel{\Xi}{0}}$ & 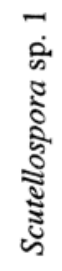 & A & B \\
\hline Anemia sp. & - & 21 & - & - & - & 21 & 1 \\
\hline Araceae & - & - & - & - & 6 & 5 & 1 \\
\hline B. serrulatum & 60 & - & 60 & - & - & 120 & 2 \\
\hline C. hirta & - & - & 9 & - & - & 9 & 1 \\
\hline E. edulis & 6 & - & - & - & - & 6 & 1 \\
\hline G. elegans & - & 9 & - & - & - & 9 & 1 \\
\hline Paspalum sp. & - & 15 & 6 & - & - & 21 & 2 \\
\hline Paspalum sp. & - & - & 9 & - & - & 9 & 1 \\
\hline P. aduncum & - & - & - & 9 & - & 9 & 1 \\
\hline P. nuda & - & - & - & - & 3 & 3 & 1 \\
\hline 19 & - & 6 & - & - & - & 6 & 1 \\
\hline 20 & - & 3 & - & - & - & 3 & 1 \\
\hline 25 & - & 6 & - & - & - & 6 & 1 \\
\hline $\mathrm{C}$ & 66 & 60 & 84 & 9 & 9 & & \\
\hline D & 2 & 6 & 4 & 1 & 2 & & \\
\hline
\end{tabular}

Convenções:

A - n. total de esporos de fungos MVA em função da espécie de planta hospedeira que teve sua rizosfera investigada

B - n. total de espécies de fungo MVA que ocorreu em cada espécie de planta hospedeira que teve sua rizosfera investigada

C - n. total de esporos de fungo MVA de cada uma das espécies verificadas

D - n. total de rizosferas de cada uma das espécies de plantas investigadas, em que ocorreu cada um dos taxa de fungo MVA. 
Tabela 6: Distribuição dos gêneros de fungos MVA em função das rizosferas das plantas hospedeiras.

Plantas

hospedeiras

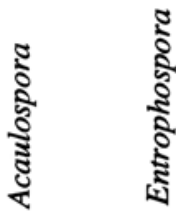

A
A B

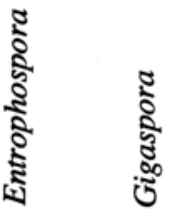

A B A
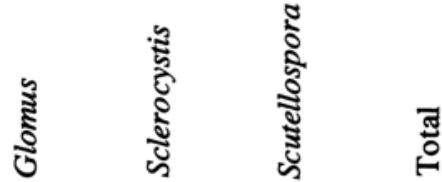

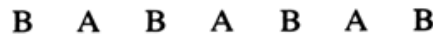

Anemia sp.

Aphellandra sp

Araceae

Begonia sp.

$B$. serrulatum

C. asiatica

C. hirta

C. cordifolium

Compositae

Cyperus sp.

D. thyrsiflora

E. edulis

G. elegans

I. affinis

L. barbinervis

Maranta sp.

N. caerulia

$P$. rigida

Paspalum sp.

$P$. aduncum

$P$. nuda

Selaginella sp.

$S$. humboldtiana

Tibouchina sp.

19 a

$19 \mathrm{~b}$

$19 \mathrm{c}$

20

23

$25 \mathrm{a}$

$25 \mathrm{~b}$

26

\section{$\begin{array}{lll}3 & 1 & -\end{array}$}

30

18

6

192

9

75

12

21

21

6

39

99

6

27

57

21

$-2----19$

$\overline{6} \overline{1}-\overline{-}-\overline{-} 21$

$31----$

- - - - - 6

$61----9$

$541---263$

$\begin{array}{lllllll}4 \overline{8} & \overline{2} & \overline{8} & \overline{1} & - & - & 30 \\ 30\end{array}$

$-\quad-\quad-\quad-12$

$\begin{array}{lllllll}11 \overline{1} & \overline{3} & \overline{30} & \overline{1} & - & - & 9 \\ & & - & & \end{array}$

$\overline{9} \overline{2}-\overline{-}-\bar{c} \quad 60$

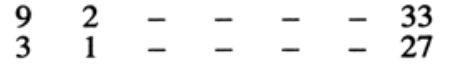

882

12

26

16,64

31

\section{8}

1

0,91

1,7 $182--\quad-\quad-114$

\section{$3 \quad 1569$}

$1 \quad 12$

0,06

0,1
29

29,09

55,3 $\overline{3}-2$

$\begin{array}{llllrr}3 & 1 & 21 & 1 & 84 & 3\end{array}$

$31--393$

- $-\quad-124$

- -1202312

$-\quad-\quad-60$

$-\quad-9$

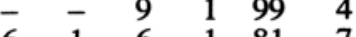

$\begin{array}{llllll}6 & 1 & 6 & 1 & 81 & 7\end{array}$

$31--243$

$31-24$

$-\quad-\quad-291$

$\begin{array}{lllll}3 & 1 & 9 & 1 & 180\end{array}$

$-\quad 212102$

$-\quad-\quad-96$

$632--141$

- $\quad-\quad-219$

- $\quad-\quad-21$

- $\quad 9127$

$\begin{array}{lllll}15 & 1 & 9 & 1 & 156\end{array}$

$\begin{array}{rrrrrr}3 & 1 & 9 & 1 & 156 & 8 \\ & 1 & 3 & 1 & 9 & 3\end{array}$

- - - 61

$-\quad--152$

$91--1265$

- $-\quad-\quad-301$

- -611025

$-\quad-\quad-121$

- 31122

$-\quad-\quad-1746$

$\begin{array}{lllllll}1 & - & - & \overline{6} & \overline{6} & 60 & 1 \\ 2 & - & - & 6 & 1 & 48 & 5\end{array}$

$2-1-\quad-\quad-303$

Convençōes:

A - n. total de esporos de fungos MVA em função da espécie de planta hospedeira que teve sua rizosfera investigada

B - n. total de espécies de fungo MVA que ocorreu em cada espécie de planta hospedeira que teve sua rizosfera investigada 
C - n. total de esporos de fungos MVA, por gênero, em todas as rizosferas investigadas

D - $\mathrm{n}$. total de espécies de fungos MVA, por gênero, que ocorreram em todas as rizosferas investigadas

E - n. total de espécies de plantas, cujas rizosferas foram investigadas, e que apresentaram esporos de fungos MVA, por gênero

F - n. de esporos de fungos MVA/100g de solo, em todas as espécies de plantas que tiveram suas rizosferas investigadas

G - porcentagem do número de esporos de fungos MVA, por gênero, em todas as espécies de plantas que tiveram suas rizosferas investigadas

Tabela 7: Resultados da análise dos elementos químicos dos solos estudados.

\begin{tabular}{|c|c|c|c|c|c|c|c|c|c|c|}
\hline \multirow{2}{*}{$\begin{array}{c}\text { Amostra } \\
\text { de solo }\end{array}$} & $\mathbf{P}$ & MO & $\mathrm{pH}$ & $\mathbf{K}$ & $\mathrm{Ca}$ & $\mathbf{M g}$ & Al & $\mathbf{S}$ & \multirow[t]{2}{*}{$\mathbf{T}$} & \multirow{2}{*}{$\begin{array}{l}\text { V } \\
\%\end{array}$} \\
\hline & $\mu \mathrm{g} / \mathrm{cm}^{3}$ & $\%$ & $\mathrm{CaCl}_{2}$ & & \multicolumn{4}{|c|}{ m. eq. $/ 100 \mathrm{~cm}^{3}$} & & \\
\hline 1 & 7 & 4,5 & 3,9 & 0,19 & 0,4 & 0,6 & 9,8 & 1,2 & 11,0 & 11 \\
\hline 2 & 7 & 4,6 & 4,5 & 0,30 & 2,1 & 1,1 & 4,1 & 3,5 & 7,6 & 46 \\
\hline
\end{tabular}

Na literatura há relatos de até oito diferentes espécies de fungos MVA em uma mesma espécie de planta que teve sua rizosfera estudada (Koske, 1987), sendo que Trufem \& Bononi (1985) verificaram 15 espécies de fungos MVA na rizosfera de cana-de-açúcar e arroz. Trufem (1988) chegou a verificar até 19 diferentes espécies de fungos MVA em rizosferas de Diodia radula Cham. \& Schlecht. (Rubiaceae) e de Smilax elastica Griseb (Smilacaceae).

Os resultados"do presente trabalho confirmam a existência de elevada diversidade de espécies de fungos MVA em uma mesma rizosfera, observação válida tanto para ecossistemas naturais como implantados (Koske, 1987; Trufem \& Bononi, 1985).

Conforme referem Mosse et al. (1981), as espécies de Glomus tem distribuição mundial, enquanto as espécies de Gigaspora/Scutellospora e Sclerocystis são mais comuns nos trópicos, sendo que as espécies de Acaulospora, embora cosmopolitas, não são predominantes.

Os presentes resultados concordam parcialmente com essas afirmações, uma vez que as espécies de Glomus efetivamente foram mais abundantes (55,3\% do total dos esporos verificados). Por outro lado, Gigaspora e Scutellospora somaram apenas $8,2 \%$ dos esporos, enquanto Sclerocystis representaram apenas 3,8\% do total de esporos. Já as espécies de Acaulospora, sugeridas como não dominantes, ocorreram com 882 esporos, ou seja, $31 \%$ do total verificado.

Os dados da Tabela 6 evidenciam ainda a existência de espécies de plantas cujas rizosferas apresentaram números diferentes de fungos MVA. Assim, B. serrulatum apresentou 312 esporos, $E$. edulis, 291, N. caerulia 219, G. elegans, 180, $P$. aduncum 156, e assim por diante, até chegar-se à Selaginella 
sp. com seis esporos, existindo ainda rizosferas que não apresentaram esporo algum como é o caso de Bauhinia sp., $P$. tristicula, $Q$. arvensis, entre outras.

As espécies de plantas das famílias Rosaceae, Gramineae, Leguminosae, Liliaceae, Malvaceae, Rubiaceae, Euphorbiaceae, entre outras, são menciónadas como altamente suscetíveis à micorrização (Mosse et al., 1981) Po de-se verificar, no entanto, que Bauhinia sp. (Leguminosae) e $P$. tristicula (Rubiaceae) não apresentaram esporos em suas rizosferas. Por outro lado, a literatura menciona como espécies pouco suscetíveis à micorrização as pertencentes às famílias Cyperaceae, Commelinaceae, Chenopodiaceae, Urticaceae, entre outras (Gerdemann, 1968). Também foram verificados esporos nas rizosferas de Cyperus sp. (Cyperaceae. 24 esporos) e de D. thyrsiflora (Commelinaceae, 36 esporos) (Tabela 6).

Esses resultados sugerem que os conceitos de suscetibilidade devem ser reavaliados, não sendo conveniente a afirmação taxativa em relação ao fenômeno. Trabalhos anteriores vêm demonstrando que esporos de fungos MVA ocorrem em rizosferas de plantas ditas não suscetíveis e vice-versa, quer sob vegetação nativa, quer sob culturas de interesse econômico (Bononi \& Trufem, 1983; Trufem \& Bononi, 1985; Trufem, 1988).

Ainda na Tabela 6, pode-se observar os valores médios estimados para o número de esporos de fungos MVA $/ 100 \mathrm{~g}$ de solo, onde se percebe que os esporos de Glomus predominam $(29,09$ esporos/100g de solo), seguindo-se os de Acaulospora $(16,64)$, Scutellospora $(4,3)$ e os de Sclerocystis, Entrophospora e Gigaspora, com valores significativamente menores.

A média do número de esporos de fungos MVA no ecossistema estudado fica, assim, em torno de 50,04 esporos $/ 100 \mathrm{~g}$ de solo, o que não pode ser considerado um número elevado, uma vez que outros autores, também para ecossistema de mata, verificaram valores variando de 11 a 384 esporos $/ 100 \mathrm{~g}$ de solo (Read et al., 1976) e de 11 a 161 esporos/100g de solo (Santos \& Vinha, 1982). Nesse sentido, pode-se verificar que o ecossistema de mata, quando comparado com ecossistemas de restinga e dunas da mesma Ilha do Cardoso (Trufem, 1988), apresentou a menor densidade de esporos no solo $(58,77$ esporos/100g de solo de duna; 192,67 esporos $/ 100 \mathrm{~g}$ de solo de restinga). Read et al. (1976), Whittinghan \& Read (1982) e Francis et al. (1986) sugerem que em mata, onde as raízes geralmente se desenvolvem como trama muito emaranhada, pode ser mais eficiente a colonização de raízes por interconecções de hifas, com o conseqüente fluxo de nutrientes. Nestes casos, a produção de número elevado de esporos pode ser encarada como desperdício de energia e matéria, uma vez que as hifas garantem a colonização de raízes de diferentes plantas hospedeiras.

No que se refere à análise dos elementos químicos (Tabela 7), os solos estudados apresentaram baixos teores de $\mathrm{P}\left(7 \mu \mathrm{g} / \mathrm{cm}^{3}\right)$, baixos teores de M.O. $(4,5$ e 4,6\%) e acidez (pH 3,9 e 4,5), com elevado teor de Al $(9,8$ e 4,1).

A literatura menciona (Hayman \& Tavares, 1985; Wang et al., 1985) 
que baixos pH favorecem as espécies de Acaulospora, Gigaspora e Scutellospora, enquanto as espécies de Glomus preferem $\mathrm{pH}$ mais próximos da neutralidade. Os dados da Tabela 6 de certa forma contrariam essas afirmaçổes, uma vez que Glomus foi bem representado em número de esporos (1569 esporos, ou 58,3\% do total), sobrepujando mesmo a somatória do número de esporos de Acaulospora, Gigaspora e Scutellospora (1113 esporos ou $39,2 \%$ do total).

Os dados presentes sugerem a existência de adaptações fisio-ecológicas dos fungos MVA aos diferentes tipos de ambientes. Nesse aspecto, a existência de ecotipos já é sugerida por Gildon \& Tinker (1983a,b), que isolaram Glomus mosseae (Nicol. \& Gerd.) Gerd. \& Trappe de regiōes sujeitas a chuvas ácidas e com metais pesados e é fato corrente, em literatura, que a espécie em questão prefere ambientes com $\mathrm{pH}$ neutros ou até mesmo levemente alcalinos.

Para finalizar pode-se perceber que algumas espécies de fungos MVA podem ser apontadas como típicas do ecossistema de mata estudada, não tendo sido verificadas com a mesma abundância em outros ecossistemas. São elas $\boldsymbol{G}$. monosporum e as espécies de Sclerocystis.

\section{Agradecimentos}

A autora agradece a pesquisadora científica Elisabete Aparecida Lopes, do Instituto de Botânica, pela identificação das plantas cujas rizosferas foram estudadas; à Seção de Fertilidade do Solo, do Instituto Agronômico, Campinas, SP, pela análise dos elementos químicos dos solos.

\section{Referências Bibliograficas}

BAREA, J.M., A.C. AZCÓN-AGUILAR, \& B. ROLDAN-FAJARDO. 1984. Avances recientes en el estudio de las micorrizas V.A. Formación, funcionamento y efectos en nutrición vegetal. An. Edaf. Agrobiol. 43:659-677.

BONONI, V.L.R. \& S.F.B. TRUFEM. 1983. Endomicorrizas vesículo-arbusculares do cerrado da Reserva Biológica de Moji-Guaçú, SP, Brasil. Rickia 10:55-84.

CABALA-ROSAND, P. \& R. DIAS. 1986. Associaçōes micorrízicas e nutrição mineral das plantas In Anais da I Reunião Brasileira sobre Micorrizas. Edições FAEPE Lavras: p. 33-59.

DAY, L.D., D.M.SYLVIA. \& M.E. COLLINS. 1987. Interactions among vesicular arbuscular mycorrhizas, soil and landscape position. Soil Science Soc. Am. J. 51:635-639.

DEHNE, H.W. 1982. Interaction between vesicular-arbuscular mycorrhizal 
fungi and plant pathogens. Phytopathol. 72:1115-1119.

FRANCIS, R., R.D. FINLAY \& D.J. READ. 1986. Vesicular-arbuscular mycorrhizas in natural vegetation systems. IV. Transfer of nutrients in inter and intra-specific combinations of host plants. New Phytol. 102:103-111.

GERDEMANN, J.W. 1968. Vesicular-arbuscular mycorrhizae and plant growth. Ann. Rev. Phytopathol. 6:397-418

GERDEMANN, J.W. \& T.H. NICOLSON. 1963. Spores of mycorrhizal Endogone species extracted from soil by wet sieving and decanting. Trans. Br. Mycol. Soc. 46:235-244.

GILDON, A. \& P.B. TINKER. 1983a. Interactions of VAM infection and heavy metal in plants. I. Effects of heavy metals on development of VAM..New Phytol. 95:241-261.

GILDON, A. \& P.B. TINKER. 1983b. Interactions of VAM infection and heavy metals in plants. II. The effects of infection on uptake of cooper. New Phytol. 93:263-268.

HAYMAN, D.S. \& M. TAVARES. 1985. Plant growth responses to vesicular-arbuscular mycorrhiza. XV. Influence of soil $\mathrm{pH}$ on the symbiotic efficency of different endophytes. New Phytol. 100:367-377.

HOGBERG, P. 1982. Mycorrhizal associations in some woodland forest trees and shrubs in Tanzania. New Phytol. 92:407-415.

JANSE, J.M. 1986. Les endophytes radicaux de quelques plantes javanaises. Ann. de Jard. Bot Buitenzorg 14:563-212.

KOSKE, R.E. 1987. Distribution of VA mycorrhizal fungi along a latitudinal temperature gradient. Mycologia 79:55-68.

KRUCKELMANN, H.W. 1975. Effects of fertilizers, soil, soil tillage and plant species on the frequency of Endogone clamydospores and mycorrhizal infection in arable soils In F.E. SANDERS, B. MOSSE \& P.B. TINKER. (ed.) Endomycorrhizas. Academic Press London: p. 511-525.

MOSSE, B., D.P. STRIBLEY. \& F. LE TACON. 1981. Ecology of mycorrizae and mycorrhizal fungi..Adv. Microb. Ecol. 5:137-210.

READ, D.J., H.K. KOUCHEKI \& J. HODGSON. 1976. Vesicular-arbuscular mycorrhiza in natural vegetation systems. I. The occurence of infection. New Phytol. 77:641-643.

REDHEAD, J.F. 1968. Mycorrhizal associations in some Nigerian forest trees. Trans. Br. Mycol. Soc. 51:377-387.

REEVES, F.B., D. WAGNER, T. MOORMAN \& J. KIEL. 1979. The role of endomycorrhizae in revegetation pratices in the semi-arid west. I. A comparison of incidence of mycorrhizae in severely disturbed vs. natural environments. Am. J. Bot. 66:6-13.

SANTOS, O.M. \& S.O. VINHA. 1982. Ocorrência de micorrizas em árvores nativas do sul da Bahia. 1. Estação Ecológica do pau-brasil. Rev. Theobroma 12:261-265. 
ST. JOHN, T.V. 1980a. A survey of mycorrhizal infection in an Amazonian rain forest. Acta Amazonica 10:527-523.

ST. JOHN, T.V. 1980b. Uma lista de espécies de plantas tropicais brasileiras naturalmente infectadas com micorriza vesicular-arbuscular. Acta Amazonica 10:229-234.

TRUFEM, S.F.B. 1988. Fungos micorrízicos vesículo-arbusculares da Ilha do Cardoso, SP, Brasil. Tese de Doutorado. Instituto de Biociências da Universidade de São Paulo. São Paulo. 358p.

TRUFEM, S.F.B. \& V.L.R. BONONI. 1985. Fungos micorrízicos vesículoarbusculares em culturas introduzidas em áreas de cerrado. Rickia 12:165-187.

WALKER. C. 1979. The mycorrhizast and the herbarium: the preservation of specimens from VA mycorrhizal studies In Program and Abstracts of the 4th. North American Conference on Mycorrhiza. Fort Collins.

WANG, G., D.P. STRIBLEY, P.B. TINKER \& C. WANG. 1985. Soil pH and vesicular-arbuscular mycorrhizas. In FITTER, A.H. ed. Ecological interactions in soil: plants, microbes and animals. Oxford: Blackwell. p. 219-224.

WHITTINGHAM, J. \& D.I. READ. 1982. Vesicular-arbuscular mycorrhiza in natural vegetation systems. III. Nutrient transfer between plants with mycorrhizal interconections. New Phytol. 90:277-284. 